\title{
Kelvin wave hydraulic control induced by interactions between vortices and topography
}

\author{
Andrew McC. $\operatorname{Hogg}^{1} \dagger$, William K. Dewar ${ }^{2}$, Pavel Berloff ${ }^{3}$ \\ and Marshall L. Ward ${ }^{1}$ \\ ${ }^{1}$ Research School of Earth Sciences, The Australian National University, Canberra, ACT 0200, Australia \\ 2 Department of Oceanography, Florida State University, Tallahassee, FL 32306-4320, USA \\ ${ }^{3}$ Department of Mathematics and Grantham Institute for Climate Change, Imperial College, London SW7 \\ 2AZ, UK
}

(Received 4 July 2010; revised 19 April 2011; accepted 8 August 2011; first published online 11 October 2011)

The interaction of a dipolar vortex with topography is examined using a combination of analytical solutions and idealized numerical models. It is shown that an anticyclonic vortex may generate along-topography flow with sufficient speeds to excite hydraulic control with respect to local Kelvin waves. A critical condition for Kelvin wave hydraulic control is found for the simplest case of a 1.5-layer shallow water model. It is proposed that in the continuously stratified case this mechanism may allow an interaction between low mode vortices and higher mode Kelvin waves, thereby generating rapidly converging isopycnals and hydraulic jumps. Thus, Kelvin wave hydraulic control may contribute to the flux of energy from mesoscale to smaller, unbalanced, scales of motion in the ocean.

Key words: geostrophic turbulence, hydraulic control, rotating flows

\section{Introduction}

The ocean energy budget comprises inputs from wind (Wunsch \& Ferrari 2004) and buoyancy forcing (Hughes, Hogg \& Griffiths 2009) at the ocean surface, which must be balanced by losses due to mixing and dissipation. While a fraction of energy input occurs at small spatial scales, the large-scale energy input is the dominant term for the ocean's interior; but dissipation and mixing occur primarily at small scales, implying that energy must be continuously transferred to these small scales if the energy budget is to be balanced. Conversion of energy to the mesoscale (with length scales of the order of the Rossby radius) is achieved through the well-known geostrophic instability processes, producing a rich mesoscale eddy field in the ocean. However, eddies follow the upscale energy transfer evolution commonly associated with 'balanced' flows (Charney 1971), where the combined constraints of conservation of energy and potential vorticity (PV) result in an inverse cascade. The implication is that energy does not reach the small scales needed for mixing and dissipation; the absence of a significant trend in mesoscale energy indicates that there must exist processes which subvert the inverse cascade and generate smaller scale, 'unbalanced' 
flow (in which a forward cascade dominates energy transfers) from the balanced flow field.

There are compelling reasons for needing to quantify the rate of energy conversion from the mesoscale eddy field. The ocean energy budget and associated mixing play an important role in setting aspects of the large-scale overturning circulation, but poor constraints on the energy budget complicate advances in this area (Ferrari \& Wunsch 2009). In particular, the mechanisms contributing to the loss of balance will control the location of mixing and dissipation. Models of the ocean circulation are now reaching resolutions at which the mesoscale flow can be at least partially resolved; but scales of mixing and dissipation remain out of reach and will continue to require parameterizations in the foreseeable future. Advanced parameterizations which correctly capture the mechanisms of energy loss from the mesoscale flow will require detailed physical understanding of these mechanisms.

Thus, the identification of loss of balance mechanisms is a topical subject in physical oceanography. Spurred by observations of elevated mixing in regions of strong mesoscale flow and rough topography (see Naveira Garabato et al. 2004), it has been proposed that topography may be one of the primary catalysts in removing balanced energy. Nikurashin \& Ferrari (2010) have predicted the generation of internal waves from mesoscale flow interacting with rough topography, while Dewar \& Hogg (2010) proposed that large-amplitude, but smooth, topography may also catalyse unbalanced flow. Molemaker, McWilliams \& Dewar (2011) found extreme vorticity generation in the Monterey Bay region, driven by interaction between the California Undercurrent and the bottom boundary layer, where flow separation and submesoscale features were generated. From the above three examples it seems that topographic interaction may provide a means of connecting balanced and unbalanced flow in the ocean.

In this paper we focus on the dynamics which leads to the generation of unbalanced flow from a balanced vortex that was recently proposed by Dewar \& Hogg (2010). This study sets out to examine whether modes of interaction between balanced vortices and large-amplitude (but not necessarily rough) topography may catalyse high-frequency, small-scale motions. A set of numerical experiments was devised in which a heton-like dipole was initialized in a continuously stratified fluid with a length scale of the order of the first Rossby radius. The dipole propagated westward (during which time it equilibrated and became essentially balanced) until it struck topography. Large-amplitude instability and overturning ensued.

An example of the vortex-topography interaction simulated by Dewar \& Hogg (2010) is shown in figures 1 and 2. Figure 1 shows Rossby number (defined by $\left.R o=\left(v_{x}-u_{y}\right) / f_{0}\right)$ averaged vertically over the depth range $713-1057 \mathrm{~m}$ in a $1600 \mathrm{~m}$ deep domain. The anticyclonic vortex is indicated by the negative (dashed) contours while the cyclonic vortex has positive Ro. As the vortex approaches the wall, both vortices feel the image effect of the wall and are pushed away from the centreline. During this process, the cyclone remains stable, while the anticyclone generates strong horizontal shear, and large $R o$ adjacent to the wall and on the northern side of the vortex. At this stage, strong overturns and mixing were observed close to the wall, while internal waves propagated into the interior of the domain (see Dewar \& Hogg 2010, for further details).

In searching for a mechanism to explain the observed phenomena, the pertinent characteristics of the instability are that it occurs only on the anticyclonic vortex; that it is maximum on the wall, while decaying exponentially away from the wall; it occurs on vertical walls, slopes and shaped seamounts (being exacerbated by shaped or 

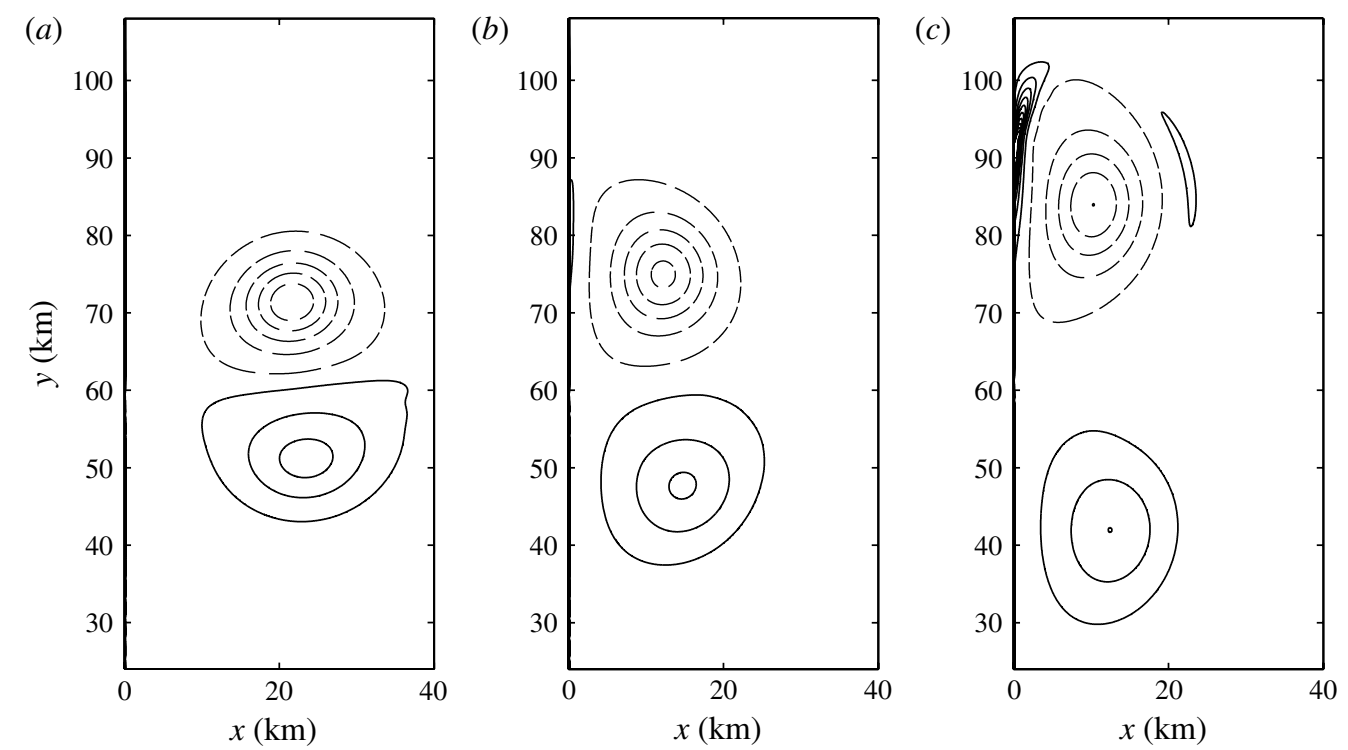

FIGURE 1. Interaction of the vortex dipole with a vertical wall, from Dewar \& Hogg (2010). Each panel shows a plan view of the Rossby number averaged across the central depths of the domain (the contour interval is 0.1 ; negative contours are shown by dashed lines). (a) At day 30 , the balanced vortex approaches the wall; $(b)$ by day 36 both vortices feel the image effect of the adjacent wall and diverge from one another; $(c)$ at day 40 there are clear signs of large, positive Rossby number on the wall adjacent to the anticyclonic vortex.

sloping topography); and that it occurs with free-slip boundary conditions. Thus, it is claimed to be an essentially inviscid phenomenon.

The asymmetry between the vortices and the exponential decay from the wall indicate a likely relationship with Kelvin waves, and this was the primary line of enquiry pursued by Dewar \& Hogg (2010). It was shown that the along-wall flow could be intensified by the interaction with the wall, and that the flow could thereby exceed the local phase speed of a higher mode Kelvin wave. This process is shown in figure 2. The evolution of isotherms adjacent to the western wall shows how the disturbance is centred around a region where northward velocities exceed $0.2 \mathrm{~m} \mathrm{~s}^{-1}$ (figure $2 b$ ). To the north of this location, isotherms evolve in such a way to converge, and thereby further accelerate flow (figure $2 c$ ) as the disturbance grows. No such effect was observed in regions where southward velocities exceed $0.2 \mathrm{~m} \mathrm{~s}^{-1}$, implying that the asymmetry is indeed due to Kelvin wave effects. Thus, it was inferred that a vortex with mode two structure interacts with topography in such a way as to arrest higher mode Kelvin waves, thereby leading to instability. The arresting of local waves was used to imply that flow may be hydraulically controlled with respect to high mode Kelvin waves in the near-wall region. Such hydraulically controlled flows are not uncommon in stratified flows interacting with topography, however, no general solution for this Kelvin wave problem exists. In particular, Dewar \& Hogg (2010) were unable to derive a control condition against which the flow state could be compared to indicate the presence or otherwise of hydraulically controlled flow. Thus, connections between the near-wall vortex instability and Kelvin wave hydraulic control remain an inference. 

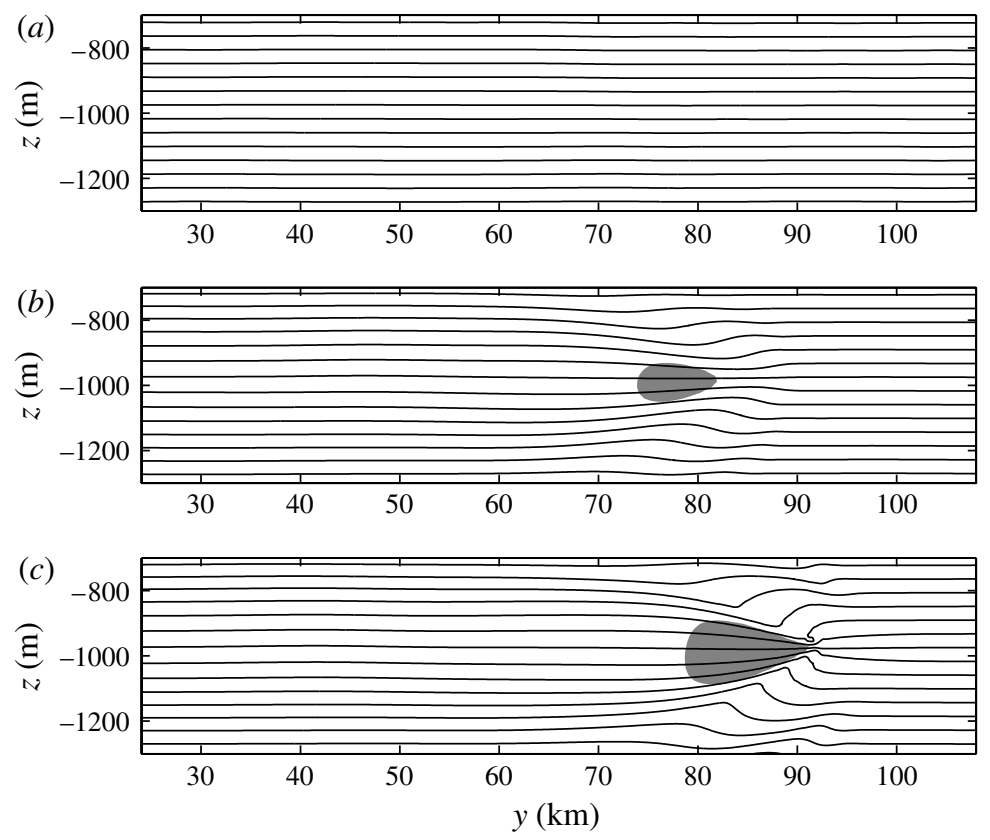

FIGURE 2. Transect of the temperature field adjacent to the western wall from the simulation shown in figure $1\left(\mathrm{CI}\right.$ is $0.1{ }^{\circ} \mathrm{C}$; shaded regions show where the northward velocity exceeds $\left.0.2 \mathrm{~m} \mathrm{~s}^{-1}\right)$. (a) Before the dipole reaches the wall at day 30, isotherms are level; $(b)$ by day 36 , large northward velocities generate perturbations in the isotherms; and (c) by day 40 these perturbations have grown to finite amplitude and generate high Rossby number flow close to the wall, as shown in figure 1. Overturning, mixing and internal wave generation ensue.

In the present paper we aim to explicitly link the dynamics first observed by Dewar \& Hogg (2010) with hydraulic control due to Kelvin waves, and thereby clarify the mechanism generating the instability. We do this by simplifying the problem to a reduced gravity, 1.5-layer case. This approach allows one-dimensional (alongwall) solutions which reveal a critical condition analogous to hydraulic control of a stratified flow. Furthermore, time-dependent and two-dimensional simulations are used to determine whether critical flow can be attained in the time scales of interaction between the vortex and topography.

The reduced gravity framework is introduced in the next section, and analytical solutions (both steady and time dependent) for the along-wall flow are given in $\S 3$. We also simulate the along-wall flow using a time-dependent model. In $\S 4$ the entire process is modelled in two dimensions (including the balanced flow impacting upon the wall). The results are discussed in $\S 5$.

\section{Shallow water framework}

The reduced gravity framework is now introduced, and simplified to provide an along-wall equation for the development of the flow excited by vortex-wall interaction. The 1.5-layer reduced gravity shallow water equations for a rotating fluid layer of depth $h$ are written as follows:

$$
u_{t}+u u_{x}+v u_{y}-f v=-g^{\prime} h_{x},
$$




$$
\begin{gathered}
v_{t}+u v_{x}+v v_{y}+f u=-g^{\prime} h_{y}, \\
h_{t}+(u h)_{x}+(v h)_{y}=0 .
\end{gathered}
$$

These equations describe motion in a layer of depth $h$ and density $\rho_{0}$ on a flatbottomed $f$-plane domain, with an infinitely deep overlying fluid of density $\rho_{0}-\Delta \rho$. We have defined $g^{\prime}=g \Delta \rho / \rho_{0}$ and other notation is standard. In what follows we assume that free-slip sidewall boundary conditions apply. To derive an evolution equation for $\mathrm{PV}, q \equiv\left(f+v_{x}-u_{y}\right) / h$, we take the time derivative of $\mathrm{PV}$ to write

$$
q_{t}+u q_{x}+v q_{y}=0 .
$$

The set of (2.1), (2.2) and (2.4) can be simplified substantially if one considers only the near-wall dynamics. Let us assume that a vortex dipole, generated in the interior of the domain, has been advected close to the western wall of the domain. This assumption is designed to match the numerical experiments of Dewar \& Hogg (2010) with some key points of departure. Since we only have a single layer (or, equivalently, a single vertical mode), the stable hetonic dipole structure (with two vortices at different vertical locations in the continuously stratified domain) is not possible in this framework, and we assume that the dipole comprises a positive and a negative vortex in the same fluid layer. On the western wall $u=0$, so that our three equations become

$$
\begin{gathered}
f v=g^{\prime} h_{x} \\
v_{t}+v v_{y}=-g^{\prime} h_{y} \\
q_{t}+v q_{y}=0 .
\end{gathered}
$$

Suppose that the interaction of the vortex with the wall can be represented by imposing a steady geostrophic background flow $v_{g}(y)$ on the wall (effectively transforming the slowly moving vortex into a stationary reference frame). Note that the superposition of the geostrophic background flow and any perturbations in the flow, $v^{\prime}(x, y, t)$, must satisfy the above equations. An immediate simplification is possible by noting that (2.7) implies $q$ is conserved along the wall, so that both the geostrophic and total PV fields satisfy the constraint

$$
\frac{f+v_{g x}}{H+h_{g}}=\frac{f+v_{g x}+v_{x}^{\prime}}{H+h_{g}+h^{\prime}}=\frac{f}{H},
$$

where $H$ is the height $h$ at some distance from the interaction region where $v=v_{x}=0$, $h_{g}$ is the perturbation to layer thickness due to the geostrophic velocity $v_{g}$ and $h^{\prime}$ the perturbation due to $v^{\prime}$. This allows us to rewrite PV conservation along the wall as

$$
h^{\prime}=H v_{x}^{\prime} / f \text {. }
$$

Equation (2.5) applies to both the geostrophic component and (hence) the perturbation part of the flow independently, so that

$$
f v^{\prime}=g^{\prime} h_{x}^{\prime} .
$$

Equation (2.10) is satisfied with a velocity field given by

$$
v^{\prime}=v_{0} e^{-x / r_{d}}
$$


where $r_{d}=c / f$ is the Rossby radius of the unperturbed flow, $c=\left(g^{\prime} H\right)^{1 / 2}$ is the baroclinic wave speed, $v_{0}(y, t)$ the perturbation velocity on the wall, and a height field

$$
h^{\prime}=h_{0} e^{-x / r_{d}}=-\left(H v_{0} / f r_{d}\right) e^{-x / r_{d}} .
$$

Note that these equations and the variables $v_{0}(y, t)$ and $h_{0}(y, t)$ are strictly defined only on the wall $(x=0)$.

We can use these constraints to write an equation for the evolution of the northward wall velocity. First, decompose (2.6) into a geostrophic and a perturbation component and substitute (2.11), (2.12) to give

$$
v_{0 t}+\left(v_{0} v_{g}+v_{0}^{2} / 2-c v_{0}\right)_{y}=-\left(v_{g}^{2}\right)_{y} / 2 .
$$

Here, the right-hand side is the geostrophic background velocity field imposed by the eddy, while the evolution of $v_{0}$ can be calculated directly. The integration of the above equation will indicate whether near-wall vortex instability occurs for a given set of prescribed conditions, and how long it takes to form (i.e. whether it can form quickly relative to the passing of the vortex).

\section{Solutions to the wall equation}

\subsection{Steady solution}

If one assumes that finite-amplitude effects can develop quickly compared with the duration of the vortex-wall interaction, then (2.13) can be viewed as steady state. In such a steady state, the evolution equation for $v_{0}$ becomes

$$
\left(v_{g}+v_{0}-c\right) v_{0 y}=-\left(v_{g}+v_{0}\right) v_{g y} .
$$

Provided that the background geostrophic velocity has a form that has a maximum in $v_{g}$ (i.e. $v_{g y}=0$ ) somewhere along the wall (presumably this will occur near the centre of the vortex), then the right-hand side of (3.1) disappears. Thus, at this point, either $v_{0 y}=0$ or else

$$
v=v_{g}+v_{0}=c .
$$

Equation (3.1) places a strong constraint on the flow, which is closely analogous to the solution for hydraulic control defined by Wood (1968) for stratified, non-rotating flows and further developed for cases of rotating flows (see Pratt \& Whitehead 2008). Traditional hydraulic solutions apply to fluids passing through a contracting channel or over large topography; the topography provides a choke point at which either interfacial slope (in the along-flow direction) is zero, or else the flow velocity exactly matches the speed of long, linear internal waves. The choke point is defined as a point of hydraulic control, and is a transition between upstream subcritical flow (i.e. the total velocity, $v$, is less than the internal wave speed, $c$ ) to downstream supercritical flow (where $v>c$ so that disturbances are advected in the direction of flow).

In the present case, the analogy with stratified hydraulic control is that the symmetry about the vortex centre provides a choke point during the vortex-wall interaction. Equation (3.1) shows that, either along-wall velocity (and thus layer thickness) is constant, or else a control condition of the form (3.2) must be met. The point of departure from the traditional hydraulic theory is that it is the speed of long, linear Kelvin waves (rather than internal waves) that governs the control point. This may seem a minor discrepancy, but it is notable that (3.2) is unlikely to be satisfied if $v_{g}$ is negative; thus an anticyclonic vortex is more likely to generate the required poleward velocities for Kelvin wave hydraulic control. 
The generation of hydraulically supercritical along-wall flow is particularly pertinent to the problem of unbalanced flow. In many stratified flows, critical conditions are associated with Kelvin-Helmholtz instability and a strong hydraulic jump (required to return the flow to subcritical conditions) at which point turbulence and mixing occur. It follows that the generation of Kelvin wave hydraulic control may produce similar behaviour and convert energy from balanced to unbalanced flows as occurred in the simulations presented by Dewar \& Hogg (2010). We now proceed to investigate whether hydraulic control can develop within the timeframe of interaction between the vortex and wall.

\subsection{Time-dependent analytical solution}

An equation such as (3.1) does not determine whether or not Kelvin wave hydraulic control will occur, because the subcritical solution remains a valid alternative flow state. For the case at hand, it appears likely that an imposed geostrophic velocity which exceeds local Kelvin wave speeds near the wall will meet the control requirements; however, the parameter regimes over which controlled flow will occur and the time to reach a controlled solution cannot be determined from (3.1) alone. Thus, we look for time-dependent solutions to this problem.

Solutions to (2.13) are possible for an analytically tractable background geostrophic velocity field, $v_{g}$, using the method of characteristics. The simplest profile we can use to illustrate this solution is the triangle wave function, which we write as

$$
v_{g}= \begin{cases}\gamma c\left(1-|y| / y_{0}\right) & \text { for }|y|<y_{0}, \\ 0 & \text { for }|y|>y_{0} .\end{cases}
$$

The background geostrophic velocity field will directly generate supercritical flow if the constant $\gamma>1$. For $\gamma<1$ the background geostrophic flow will be subcritical, but the addition of perturbation velocities may be sufficient to generate critical conditions. To solve this problem using the method of characteristics we define the parameter $s$ such that

$$
\begin{gathered}
\frac{\mathrm{d} t}{\mathrm{~d} s}=1, \\
\frac{\mathrm{d} y}{\mathrm{~d} s}=v_{g}+v_{0}-c, \\
\frac{\mathrm{d} v_{0}}{\mathrm{~d} s}=-\left(v_{g}+v_{0}\right) v_{g y} .
\end{gathered}
$$

Restricting ourselves to the part of the domain where the background flow is non-zero (i.e. $|y|<y_{0}$ ) we find

$$
\begin{gathered}
\frac{\mathrm{d} y}{\mathrm{~d} s}=\left(\gamma-1-\gamma|y| / y_{0}\right) c+v_{0}, \\
\frac{\mathrm{d} v_{0}}{\mathrm{~d} s}=\operatorname{sgn}(y)\left(\gamma c\left(1-|y| / y_{0}\right)+v_{0}\right) \gamma c / y_{0} .
\end{gathered}
$$

These two equations can be combined by writing

$$
\frac{\mathrm{d}}{\mathrm{d} s}\left(v_{0}-\gamma c|y| / y_{0}\right)=\operatorname{sgn}(y) \gamma c^{2} / y_{0} .
$$


Assuming initial conditions of the form $s=0, t=0, v_{0}=0$ and $y=r$, we then integrate (3.9) to obtain

$$
v_{0}=\gamma c(|y|-|r|+\operatorname{sgn}(y) c s) / y_{0},
$$

which is an explicit equation for velocity as a function of position. Equation (3.10) is written assuming that $y$ does not change sign; if a sign change occurs, the equation becomes a little more algebraically tedious. Combining (3.7) and (3.10), we can write an equation for the evolution of $y$,

$$
\frac{\mathrm{d} y}{\mathrm{~d} s}=\left(\gamma-1-\gamma|r| / y_{0}\right) c+\operatorname{sgn}(y) \gamma c^{2} s / y_{0},
$$

which is integrated to give

$$
y=r+\left(\gamma-1-\gamma|r| / y_{0}\right) c t+\operatorname{sgn}(y) \gamma c^{2} t^{2} / 2 y_{0},
$$

where (3.4) has been used to reinstate the time variable. Equation (3.12) is an explicit equation for the trajectory of characteristics, while velocity along each characteristic can be found using (3.10).

The method of characteristics solution has some interesting features which require further investigation. First, we search for locations at which the critical condition (3.2) is met. Writing the total wall velocity from (3.3) and (3.10),

$$
v_{g}+v_{0}=\gamma c\left(1-|r| / y_{0}+\operatorname{sgn}(y) c t / y_{0}\right),
$$

we can find the time at which flow along a given characteristic becomes critical,

$$
t=\left(y_{0} / \gamma-y_{0}+r\right) / c
$$

(where we have restricted ourselves to $y>0, r>0$ for convenience). It can be seen that for $\gamma>1$ there are locations at which the flow is always supercritical, while characteristics that are initially subcritical may evolve to exceed critical conditions. For example, figure 3(a) shows the evolution of characteristics with time for a case with $\gamma=1.5$, meaning that characteristics emanating from a region close the centre of the domain are always supercritical: the boundary of this region is shown by the large black dot at $t=0$. As the system evolves, the transition to supercritical flow is marked by a small black dot on the characteristic. At this point, the characteristic line, which was initially directed against the direction of the background geostrophic flow (but in the direction of Kelvin wave propagation) is arrested by the combination of background geostrophic flow and the evolving ageostrophic velocity, and is advected back to the north. Furthermore, this case contains characteristics which propagate from negative to positive $y$, induced principally by the geostrophic background flow.

Figure 3(b) shows a case in which the background geostrophic flow is subcritical $(\gamma=0.75)$ and some characteristics propagate from positive to negative $y$. However, a number of characteristics are arrested by the flow (again, control points are shown by black dots) and returned downstream. The first characteristic to become critical does so at $y \approx 0$ (where $v_{g y} \approx 0$ ), in agreement with (3.1), and at time 0.15 days. This behaviour is robust, provided that $\gamma>0.5$; for cases with $\gamma<0.5$ no characteristics initialized in the range $|y|<y_{0}$ can be arrested.

The second key feature of the method of characteristics solution is the potential for characteristics to converge. This can be shown by searching for insensitivity of $y$ to initial conditions, i.e. the derivative of (3.12) with respect to $r$,

$$
y_{r}=1-\operatorname{sgn}(y) \gamma c t / y_{0}=0,
$$



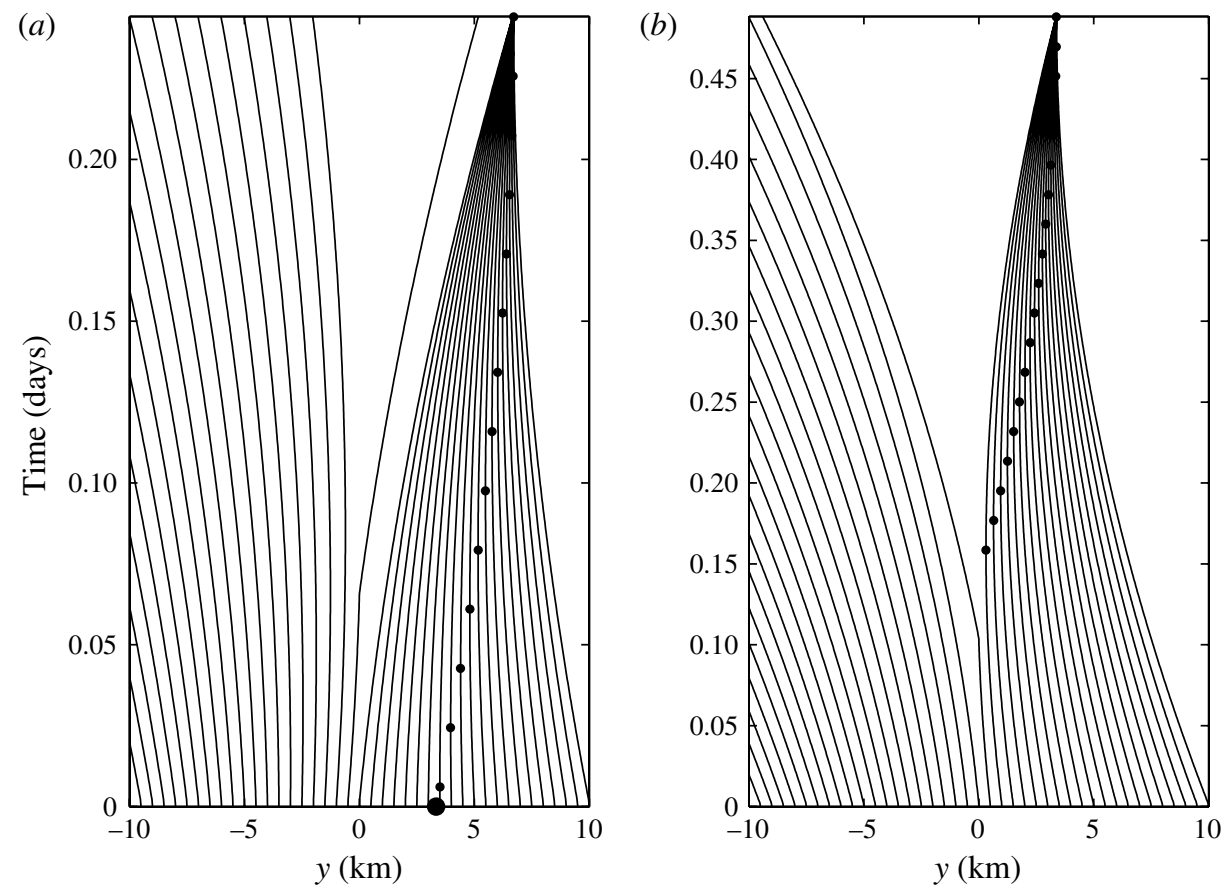

FIGURE 3. Method of characteristics solutions of (3.12) for a range of initial values of $y_{0}$, indicating the development of controlled along-wall flow. Lines track the evolution of characteristics; those lines crossing through $y=0$ are estimated by integrating (3.11). (a) Case with supercritical background geostrophic flow $(\gamma=1.5)$. The large black dot shows the point at which flow is initially critical, while the small black dots indicate the transition to supercritical flow along the characteristic. (b) Case with subcritical geostrophic flow $(\gamma=0.75)$; points at which the critical condition is met are shown by black dots.

placing a condition on the time it takes for characteristics to converge. At time $t=y_{0} / c$ characteristics stemming from the region of positive $y$ may converge. This convergence of characteristics indicates the development of a front in the flow, and is consistent with a traditional hydraulic jump required to return flow from supercritical to subcritical conditions. The location and the time to form the front depend strongly upon the value of the background geostrophic flow, with high values of $\gamma$ producing fronts more quickly and in a more northwards position. The fronts are a particular feature of both cases shown in figure 3; after the front forms the solution is no longer valid in the frontal region.

The analytical solution derived here is useful for demonstrating the conditions under which critical flow occurs. Most notably, it is shown that an imposed geostrophic flow which is subcritical everywhere can lead to the generation of supercritical flow through nonlinear evolution of the along-wall flow. In such cases, flow with a fully developed hydraulic jump occurs on time scales of the order of a day, which is sufficiently short that it is feasible for the development of Kelvin wave hydraulic control during the interaction of a baroclinic vortex with topography (a process which lasts for 5-10 days). However, the analytical solution is necessarily restricted to cases with a simple background geostrophic velocity field. Therefore, we now proceed to directly integrate (2.13) numerically to demonstrate that the emergent behaviour generating 

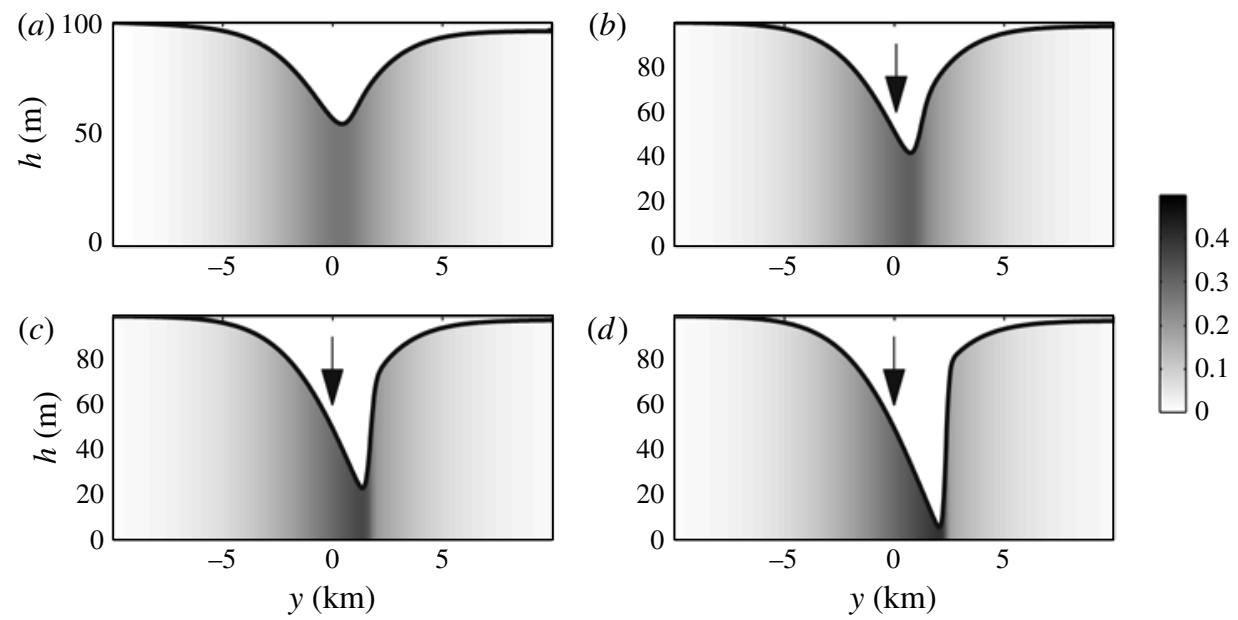

FIGURE 4. Evolution of (2.13). Greyscale shows northward velocity $\left(\mathrm{m} \mathrm{s}^{-1}\right)$ and the thick black line shows total layer thickness. Snapshots are shown at daily intervals, with the hydraulic control condition shown as a vertical arrow in cases where it is exceeded. It is clear that the front downstream of the maximum velocities occurs only after the critical condition is exceeded on day 2. (a) Day 1, (b) day 2, (c) day 3 and $(d)$ day 4.

Kelvin wave hydraulic control persists for cases with arbitrary background geostrophic velocity fields.

\subsection{Time-dependent numerical simulation}

We solve (2.13) numerically for a prescribed geostrophic flow $v_{g}(y)=\gamma c \operatorname{sech}^{2}(y / L)$ where $L=5 \mathrm{~km}, \gamma=0.5, H=100 \mathrm{~m}$ and $g^{\prime}=0.001 \mathrm{~m} \mathrm{~s}^{-2}$. Thus, the expected steady solution (see (3.1)) has velocity $v_{g}+v_{0}=c \approx 0.316 \mathrm{~m} \mathrm{~s}^{-1}$ at the eddy centre. A small viscosity $\left(K=10 \mathrm{~m}^{2} \mathrm{~s}^{-1}\right)$ is added to this equation for numerical stability. The numerical method uses a simple second-order finite difference scheme with leapfrog time stepping, where solutions are mixed every 100 time steps to prevent numerically diverging solutions. Fine spatial resolution $(33 \mathrm{~m})$ in the along-flow direction is specified.

Figure 4 shows this system evolving over 4 days. Total northwards velocity, $v=v_{g}+v_{0}$, is indicated by the greyscale, while the layer height $h=H+h_{0}$ is shown by the thick solid line. The initial state has $h_{0}=0$; velocities and layer heights then evolve to be symmetric about the centre of the eddy. Figure 4(a) shows this symmetric state after day 1 , and it is notable that the condition $v_{y}=h_{y}=0$ (the subcritical branch of (3.1)) appears to be satisfied at $y=0$. After this time, hydraulic control is initiated (at locations indicated by the black arrows) and the streamwise gradient in layer depth at $y=0$ becomes finite. By day 2 (figure $4 b$ ) the asymmetry about the centre is clearly established, and the Kelvin wave hydraulic control point is close to $y=0$. After this time, both layer height and velocity at the centre remain steady (maximum velocity of $0.315 \mathrm{~m} \mathrm{~s}^{-1}$ ), while velocities continue to grow downstream. By day 4 a strong front (hydraulic jump) is established downstream of the eddy centre.

The numerical solution is therefore broadly consistent with insights gained from the analytical solution using the method of characteristics. Both indicate the development of Kelvin wave hydraulic control in the along-wall direction, which emerges within 0.5-2 days of initiation and a hydraulic jump on the downstream side. However, the 
present results are necessarily limited by the restriction to the solution of the flow adjacent to the wall. It remains to be shown that an impinging dipole may generate the proposed hydraulic control points for the reduced gravity case; we proceed to outline two-dimensional reduced gravity shallow water simulations which attempt, as far as possible, to mimic the fully stratified vortex-topography interactions of Dewar \& Hogg (2010).

\section{Shallow water simulation}

The above arguments suggest that near-wall vortex instability should be present in a reduced gravity setting, and show that Kelvin wave hydraulic control is the physical mechanism leading to instability. However, the link between these idealized one-dimensional solutions and the fully stratified cases of Dewar \& Hogg (2010) is tenuous, unless it can be established that along-wall geostrophic flow of sufficient magnitude can feasibly be instigated by vortex-topography interactions. We now simulate the interaction of a vortex dipole with a wall using a two-dimensional 1.5layer shallow-water model. The 1.5-layer system will enable analysis of the hydraulic control condition (3.2), an interpretation which is not possible in the continuously stratified cases shown in figures 1 and 2, because a continuously stratified control condition is not currently known for this problem. Nonetheless, there remain several difficulties in modelling this flow; in particular the reduction to a single vertical mode means that the impinging vortex must necessarily be large in amplitude in order to excite Kelvin wave hydraulic control in the along-wall flow. This is in contrast to the continuously stratified case simulated by Dewar \& Hogg (2010), in which a baroclinic mode two vortex interacts with higher mode Kelvin waves. Correspondingly, asymmetries in layer depth between the anticyclone and the cyclone make it difficult to initialize a stable dipole.

The simulation is performed using GOLD, an isopycnal ocean model (a revised version of the Hallberg Isopycnal Model as described by Hallberg \& Gnanadesikan 2006). The model is on an $f$-plane in a rectangular domain of size $160 \mathrm{~km} \times 128 \mathrm{~km}$, with Coriolis parameter $f=10^{-4} \mathrm{~s}^{-1}$, reduced gravity $g^{\prime}=10^{-3} \mathrm{~m} \mathrm{~s}^{-1}$, and mean depth $H=100 \mathrm{~m}$, so that the corresponding deformation radius of $r_{D} \approx 3 \mathrm{~km}$. Advection is discretized according to the energy-conserving scheme of Sadourny (1975), while mass transport is computed using a PPM-based finite volume method (Lin et al. 1994). Time steps are computed using a second-order accurate Runge-Kutta method (Matsuno 1966). The spatial resolution is $\Delta x=100 \mathrm{~m}$ and the time step size is $\Delta t=150 \mathrm{~s}$. A very small biharmonic viscosity of $A_{4}=10^{-2} \mathrm{~m}^{4} \mathrm{~s}^{-1}$ and quadratic bottom drag of form $-C_{d}|\mathbf{u}|^{2}$ with drag coefficient $C_{d}=10^{-8}$ were used to stabilize the nonlinear transfer to small scales. Errors were bounded by the time step size $\Delta t$ and were insensitive to our choice of $A_{4}$ and $C_{d}$.

The model is initialized with a large-amplitude $f$-plane modon (a circular dipolar vortex; see Larichev \& Reznik 1976 generating interfacial displacements of the order of the layer thickness. The system is allowed to evolve for 50 days before interaction with the wall; layer thickness after this time is shown in figure 5(a). The large amplitude of the dipole and subsequent asymmetry of the vortices is immediately apparent from this figure, with thickness anomaly variations of $\pm 80 \mathrm{~m}$. The vortices are less stable than the smooth hetonic structure shown in figure 1, but after 50 days the dipole is close to a balanced state, enabling observation of the flow induced by vortex-wall interaction. 

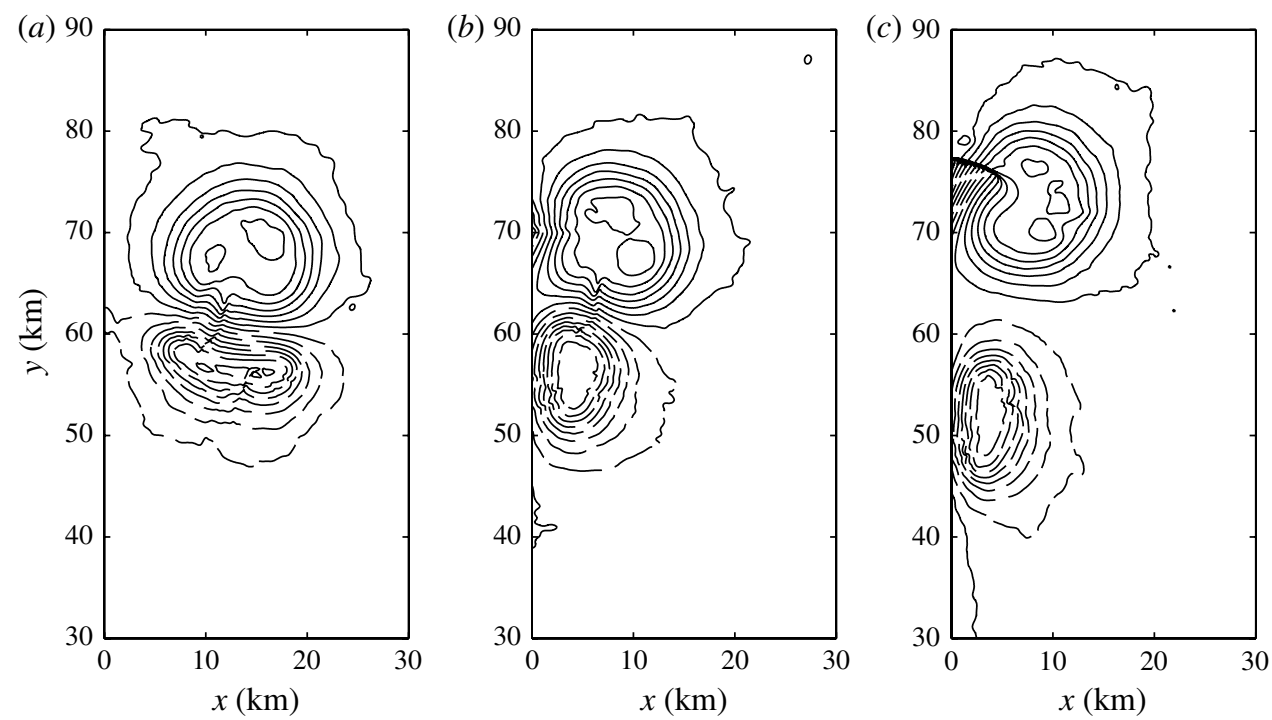

FIGURE 5. Shallow water vortex dipole interaction with the wall. Layer thickness anomalies are shown after (a) 50 days; (b) 54 days and (c) 56 days (CI $10 \mathrm{~m}$; dashed contours negative). The instability is seen close to the wall on day 56 at $y \approx 75 \mathrm{~km}$.

The interaction of the dipole with the wall proceeds in a similar way to that shown in figure 1. By day 54 (figure $5 b$ ) a small instability appears adjacent to the wall at the approximate latitude of the centre of the anticyclone, in the form of negative layer thickness anomalies. By day 56 (figure $5 c$ ) a wedge-shaped region of instability forms on the downstream side of the anticyclone, as well as a strong front. In contrast, no instability occurs near the cyclonic vortex.

It remains to demonstrate that flow in the along-wall direction matches the prediction of the one-dimensional solutions. Specifically, we examine the along-wall flow and layer thickness on the western edge of the domain, in figure 6, using the same methodology as figure 4. Initially, layer thickness is roughly constant and velocity small, but these are modified as the vortex nears the wall. By day 54 (figure $5 d$ ) strong northward and southward velocities occur, approaching Kelvin wave speeds. However, only the northward flow can satisfy the Kelvin wave hydraulic control condition (3.2): this occurs at day 55 and 56 (figure $5 e$ and $f$ ) and control points are indicated by the vertical arrow. The supercritical flow is accompanied by a dramatic decrease in layer thickness on the downstream side of the anticyclone and a return to subcritical conditions in the form of a hydraulic jump. The flow in this region is phenomenologically similar to the instability shown in figure $2(c)$, except that in the present case the location of the critical transition can be precisely located.

\section{Discussion and conclusions}

This paper is designed to demonstrate that interaction between balanced flow and topography can generate small-scale, unbalanced flow which is more easily dissipated. We use the particular example of a vortex dipole interacting with a vertical wall. However, the dynamical ingredients required for unbalanced flow are merely stratification and along-topography flow of sufficient magnitude. It follows that the mechanism proposed here applies to any topographic shape (this assertion is strongly 


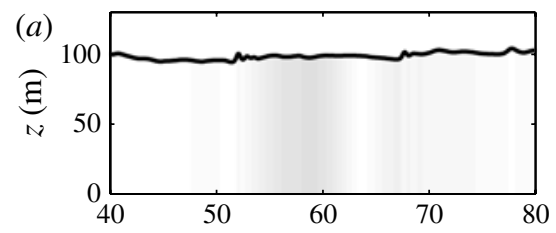

(b)
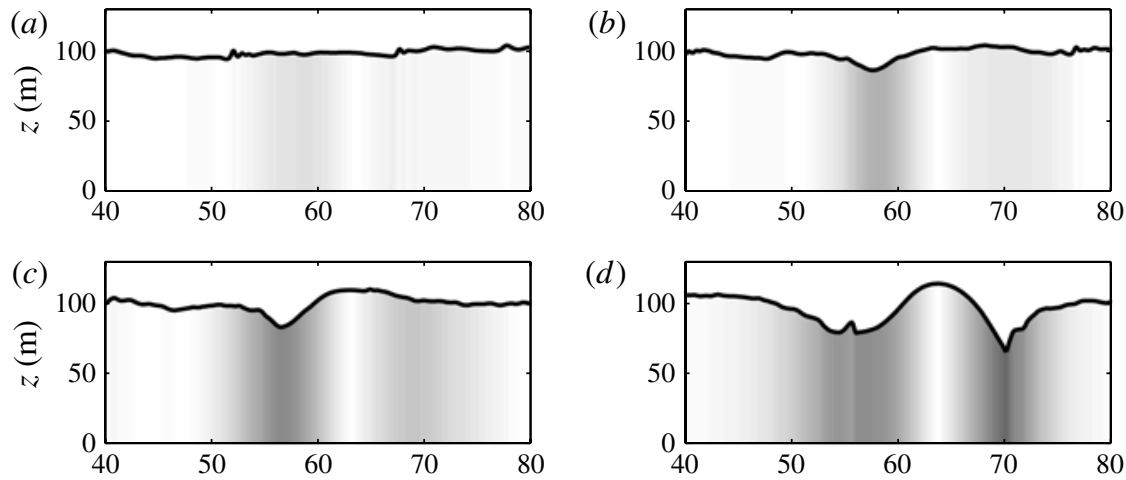

(d)
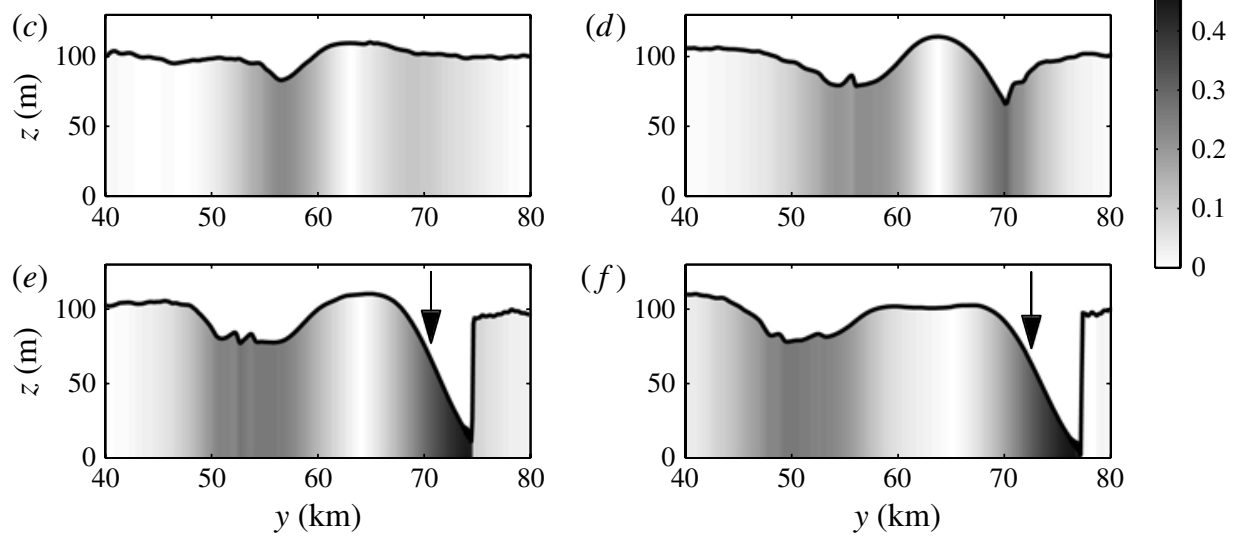

FIGURE 6. Snapshots of absolute velocity in the meridional direction (greyscale) and layer thickness (solid black line) along the western wall of the shallow water model. The vertical arrow in panels $(e, f)$ indicates where the hydraulic condition (3.2) is satisfied. (a) Day 51, $(b)$ day 52, (c) day 53, $(d)$ day 54, $(e)$ day 55 and $(f)$ day 56.

supported by the simulations of Dewar \& Hogg 2010) and to other inertial background flow types such as western boundary currents and the Antarctic Circumpolar Current. Thus, we propose that the mechanism illustrated here is robust, and may apply to many different situations in the ocean.

We have shown that vortex-topography interaction can generate flow which is supercritical with respect to local Kelvin waves, and thereby produce unbalanced flow. We have chosen to operate in a 1.5-layer reduced gravity framework, so that the single Kelvin wave mode available allows specific definition of a Kelvin wave hydraulic control condition. This condition (3.2) proves to be relatively simple to derive, and both analytical and numerical solutions indicate that the development of critical flow occurs on time scales substantially shorter than the duration of vortex interaction with the wall. The asymmetric nature of Kelvin waves means that velocities associated with an anticyclonic vortex are more likely to meet the critical condition. Finally, the shallow water simulation in $\S 4$ shows that Kelvin wave hydraulic control can be induced by a vortex dipole interacting with a vertical wall, producing strong northward acceleration and interface slopes, with a sharp jump in the lee of the vortex. These features are similar in all respects to the near-wall vortex instability phenomena simulated by Dewar \& Hogg (2010) for the case of a balanced hetonic dipole interacting with topography in a continuously stratified fluid. We therefore conclude that, despite the lack of a clear critical condition in the continuously stratified case, Kelvin wave hydraulic control is a useful analogy for the instability catalogued in Dewar \& Hogg (2010).

A significant weakness in the shallow water simulations presented in figures 5 and 6 is that the single vertical mode in the system necessitates very large amplitudes in interface displacement. This is not the case for continuously stratified flow, where 
higher Kelvin modes are available, enabling a low mode vortex to interact with these high vertical mode Kelvin waves. It follows that a criterion for the development of near-wall vortex instability in the continuously stratified case must be some function of the vertical structure. The development of such a criterion is likely to be related to conditions for Kelvin wave hydraulic control, but the additional complexities of continuous stratification and shear appear to prohibit a simple control condition.

The difficulty of finding a control condition for the continuously stratified case raises the question of whether Kelvin wave hydraulic control can occur during eddy-topography interactions in the ocean. In particular, are geostrophic velocities near the ocean floor sufficient to invoke hydraulic control? The answer to this question depends upon the modal structure and phase speed of boundary waves in the ocean and is well beyond the scope of the present paper. However, the reduced gravity in the shallow water simulations was chosen to be sufficiently small as to mimic the behaviour of higher mode waves in the ocean; we find that interior eddy velocities on the order of $10 \mathrm{~cm} \mathrm{~s}^{-1}$ (amplified to $20 \mathrm{~cm} \mathrm{~s}^{-1}$ upon topographic interaction) are sufficient to invoke controlled flow. Thus, we argue that the required geostrophic velocities are sufficiently small to make this phenomena worthy of further investigation in ocean observations and ocean models.

If it does occur in the ocean, then this instability has the potential to $(a)$ control the location and rate of mixing and dissipation in the ocean due to injection of energy from the mesoscale to smaller scales at precise locations, and $(b)$ modify the rate of loss of energy from the ocean mesoscale. An important distinguishing feature of the present instability is that it will occur for both rough and smooth topography, while other proposed mechanisms for loss of balance require rough topography (Nikurashin \& Ferrari 2010). It follows that eddy-resolving ocean models are likely to require parameterization for this mechanism, and for other contenders for the generation of submesoscale flow (e.g. Nikurashin \& Ferrari 2010; Molemaker et al. 2011). Quantifying the relative importance of these different proposed mechanisms leading to loss of balance is the subject of ongoing work on this topic.

This work was supported by an ARC Discovery Project (DP0986244), and numerical computations were conducted using the National Facility of the Australian National Computational Infrastructure. WKD is supported through NSF grants OCE0550139 and OCE-0961485. Our thanks to Bob Hallberg from GFDL for the use of the GOLD ocean model.

\section{REFERENCES}

Charney, J. G. 1971 Geostrophic turbulence. J. Atmos. Sci. 28 (6), 1087-1095.

Dewar, W. K. \& HogG, A. M. 2010 Topographic inviscid dissipation of balanced flow. Ocean Modell. 32 (1-2), 1-13.

FERRARI, R. \& WUNSCH, C. 2009 Ocean circulation kinetic energy: reservoirs, sources, and sinks. Annu. Rev. Fluid Mech. 41 (1), 253-282.

Hallberg, R. \& GNAnAdesikan, A. 2006 The role of eddies in determining the structure and response of the wind-driven Southern Hemisphere overturning: initial results from the Modelling Eddies in the Southern Ocean project. J. Phys. Oceanogr. 36, 3312-3330.

Hughes, G. O., Hogg, A. M. \& Griffiths, R. W. 2009 Available potential energy and irreversible mixing in the meridional overturning circulation. J. Phys. Oceanogr. 39, 3130-3146.

LARICHEV, V. D. \& REZniK, G. M. 1976 Strongly nonlinear two-dimensional solitary Rossby waves. Oceanology 16, 961-967. 
Lin, S.-J., ChaO, W. C., Sud, Y. C. \& Walker, G. K. 1994 A class of the van Leer-type transport schemes and its application to the moisture transport in a general circulation model. Mon. Weath Rev. 122, 1575-1593.

Matsuno, T. 1966 Numerical integrations of the primitive equations by a simulated backward difference method. J. Meteor. Soc. Japan, Ser. II 44, 76-84.

Molemaker, J. M., McWilliams, J. C. \& Dewar, W. K. 2011 Submesoscale generation of mesoscale anticyclones in the California undercurrent. J. Phys. Oceanogr. (submitted).

Naveira Garabato, A. C., Polzin, K. L., King, B. A., Heywood, K. J. \& Visbeck, M. 2004 Widespread intense turbulent mixing in the Southern Ocean. Science 303 (5655), 210-213.

Nikurashin, M. \& FerRaRi, R. 2010 Radiation and dissipation of internal waves generated by geostrophic motions impinging on small-scale topography: theory. J. Phys. Oceanogr. 40 (5), $1055-1074$.

Pratt, L. J. \& Whitehead, J. A. 2008 Rotating Hydraulics: Nonlinear Topographic Effects in the Ocean and Atmosphere. Springer.

SADOURNY, R. 1975 The dynamics of finite-difference models of the shallow-water equations. J. Atmos. Sci. 32, 680-689.

Wood, I. R. 1968 Selective withdrawal from a stably stratified fluid. J. Fluid Mech. 32, 209-223.

Wunsch, C. \& FERrari, R. 2004 Vertical mixing, energy, and the general circulation of the oceans. Аnnu. Rev. Fluid Mech. 36, 281-314. 